\title{
JOSEPHSON JUNCTION DETECTOR FOR ASTRONOMICAL APPLICATIONS
}

\author{
B. T. ULRICH
}

Department of Astronomy and Department of Physics

University of Texas, Austin, Texas 78712, USA

\begin{abstract}
Résumé. - Un détecteur large bande, dans la fenêtre de transmission atmosphérique de 1,0 à $1,6 \mathrm{~mm}$ a été développé pour des observations astronomiques au télescope optique de $2,7 \mathrm{~m}$ de l'Observatoire McDonald. Pour l'observation de sources astronomiques à spectre continu, le facteur de qualité significatif, la «Température Equivalente de Bruit » est actuellement de 0,25 K pour une seconde d'observation. Parmi les objets astronomiques observés, citons le soleil, la lune, Vénus, Jupiter, 3C 273, et le centre galactique. Avec la sensibilité actuelle, une source de longueur d'onde $1 \mathrm{~mm}$ et de 0,7 unité de flux, donnerait un rapport signal sur bruit de 1 pour une observation de $3 \mathrm{~h}$ au télescope NRAO de $11 \mathrm{~m}$. Le détecteur utilise une jonction Josephson constituée par une pointe de niobium en contact avec une pièce d'étain. La jonction est montée coaxialement dans une cavité cylindrique multimodes de $5 \mathrm{~mm}$ de diamètre, couplée par un cône à un guide d'onde circulaire de $5 \mathrm{~mm}$ de diamètre, débouchant au sommet du dewar. Le rayonnement incident de la source astronomique à spectre continu, provoque une diminution du courant critique de la jonction (c'est un phénomène à large bande). La jonction est polarisée par une résistance de source de $20 \Omega$, et on mesure la variation de la tension de polarisation due au changement du courant critique. L'interruption du rayonnement incident est fait de deux façons différentes. Pour des sources astronomiques brillantes, telles que le soleil ou la lune, un hacheur ouvre et ferme alternativement l'entrée du détecteur. Pour des sources faibles, un hacheur différentiel commute la source et le fond du ciel.
\end{abstract}

Abstract. - A wide-band detector for the 1.0 to $1.6 \mathrm{~mm}$ atmospheric transmission window has been developed for astronomical observations at the McDonald Observatory $2.7 \mathrm{~m}$ optical telescope. For observations of continuum astronomical sources, the relevant figure of merit is the "Noise

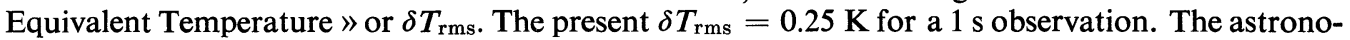
mical objects which have been observed so far include the sun, moon, Venus, Jupiter, 3C 273 and the galactic center. With the present detector sensitivity, a $1 \mathrm{~mm}$ source of 0.7 flux units would give a signal to noise of one for a $3 \mathrm{~h}$ observation at the NRAO $11 \mathrm{~m}$ telescope. The detector uses a point contact Josephson junction, with a niobium point against tin. The junction is mounted coaxially in a multimode $5 \mathrm{~mm}$ diameter cylindrical cavity which is coupled by a cone to a $5 \mathrm{~mm}$ oversize circular wave guide leading up the neck of the dewar. The wide-band mode of operation is used, in which the incident continuum radiation from an astronomical source causes a depression of the junction critical current. The junction is biased with a $20 \Omega$ source resistance, and the change in bias voltage due to the change in the junction critical current is measured. Two modes are used to chop the $1 \mathrm{~mm}$ radiation incident on the junction. For bright astronomical sources such as the sun and the moon, a total power chopper at the entrance of the wave-guide alternately opens and closes the radiometer entrance. For faint sources, a differential chopper chops between the source and adjacent sky.

1. Introduction. - We have developed a wide-band Josephson junction radiometer for the 1.0 to $1.6 \mathrm{~mm}$ spectral region, and have used it to make astronomical measurements [1], [2] at the $2.7 \mathrm{~m} \mathrm{McDonald} \mathrm{Obser-}$ vatory optical telescope, first in March, 1969. Three versions of the radiometer have been built to test different aspects of the techniques involved in using a Josephson junction to make $1 \mathrm{~mm}$ astronomical observations. The objects that have been observed so far are the sun, moon, Venus, Jupiter, 3C 273, and the Galactic Center [2], [3]. The present sensitivity is $\delta T_{\mathrm{rms}}=0.25 \mathrm{~K}$ for a $1 \mathrm{~s}$ observation at $1.2 \mathrm{~mm}$ wavelength. $A 3 \mathrm{~h}$ observation at the NRAO $11 \mathrm{~m}$ telescope with the radiometer at its present sensitivity would give an rms noise limit of 0.7 flux units $\left(1 \mathrm{fu}=10^{-26} \mathrm{~W} \cdot \mathrm{m}^{-2} \cdot \mathrm{Hz}^{-1}\right)$. This sensitivity is more than adequate to observe a large number of astronomical sources.

The Josephson junction wide-band radiometer is still in a stage of active development. Accordingly, the present level of performance should not be taken as the final criterion of its usefulness. As discussed below, the present sensitivity is limited primarily by interference from external electrical noise. It is difficult to calculate a meaningful ultimate sensitivity for a Josephson junction radiometer, and this calculation is not attempted here.

In this paper we present first the astronomical observing possibilities, then outline the features of the three Josephson junction radiometers, and finally 
describe in more detail the present radiometer from a solid state physics viewpoint and from an astronomical viewpoint.

2. Astronomical observing possibilities. - Although there is rapidly increasing astronomical activity at both millimeter (3-9 $\mathrm{mm})$ and infrared (2-350 $\mu \mathrm{m})$ wavelengths, very little successful work has been done yet at wavelengths near $1 \mathrm{~mm}$. This is no doubt due to the fact that in this intermediate region of the spectrum neither the coherent techniques commonly used at longer radio wavelengths nor the incoherent techniques (i. e. bolometers) used at infrared wavelengths are able to give adequate sensitivity. The use of Josephson junction detectors offers a tremendous potential to investigate this important and hitherto essentially unknown part of the electromagnetic spectrum.

Probably the most pressing problem to be investigated is the emission from objects previously observed to be anomalously strong in the $20-100 \mu \mathrm{m}$ far infrared. In no cases are there evidences of enhanced emission at the shortest radio wavelength previously investigated $(3 \mathrm{~mm})$ [4]. Observations at $1 \mathrm{~mm}$ are needed to study the cross-over region between the strong far infrared emission and the more usual radio emission. Observations of the intensity and of possible variations in intensity will help to discriminate between the various radiation mechanisms proposed, and in particular, help to discriminate between thermal and non-thermal processes.

Models of variable radio sources usually invoke expanding clouds of relativistic electrons in a magnetic field. The electrons radiate by the synchrotron mechanism. $1 \mathrm{~mm}$ data on known variable radio sources is essential to investigate the expanding electron cloud near the time of its origin. Although the general class of expanding cloud models adequately explains the data at the $\mathrm{mm}$ and $\mathrm{cm}$ wavelengths, the simplest model of uniform adiabatic expansion with conservation of magnetic flux is clearly inadequate [5]. The difference between models is greatest at the shorter wavelength, and $1 \mathrm{~mm}$ is probably the shortest wavelength to which the radio variations can be traced. Simultaneous observations at several frequencies of outbursts from active radio sources should give information about the expanding cloud of relativistic electrons. The flux observed at infrared wavelengths does not appear to be a simple extension of the radio synchrotron spectrum.

The nucleus of our galaxy is known to emit strongly at $100 \mu \mathrm{m}$. Observations of the nucleus of our galaxy and of other nearby galaxies at $1 \mathrm{~mm}$ may provide information about the nature of the radiation mechanism responsible for the $100 \mu$ peak.

Recent sky surveys at $100 \mu \mathrm{m}$ wavelength have produced lists of objects, some of which are not yet identified, and others identified with HII regions.
These objects can be observed to search for $1 \mathrm{~mm}$ emission.

Equally important, although perhaps less spectacular are $1 \mathrm{~mm}$ observations of the thermal emission from the planets. Even with the sensitivity now available with the present Josephson junction detector system all the planets, with the exception of Pluto, will be observable with the NRAO 36 foot telescope, and even Pluto $(0.1 \mathrm{fu})$ is not beyond sensitivities expected in the future. In the case of the nearly airless planets Mercury and Mars, $1 \mathrm{~mm}$ observations give valuable data on surface properties, while observations of Venus, Jupiter, Saturn, Uranus, and Neptune can be used to investigate atmospheric properties. These ground-based observations are an invaluable (and relatively very inexpensive) adjunct to the interpretation of planetary fly-by data obtained by space probes.

Enhanced $1 \mathrm{~mm}$ emission above regions of solar activity was observed during the first observing runs with the Josephson junction detector obtained at the $2.7 \mathrm{~m} \mathrm{McDonald}$ Observatory optical telescope [1] in April 1969. Recently, emission peaks have been reported by the Queen Mary College group who used a $1.5 \mathrm{~m}$ telescope located in London [6]. With the $2.7 \mathrm{~m}$ McDonald Observatory telescope, and with the $11 \mathrm{~m}$ Kitt Peak telescope, it is possible to obtain higher angular resolution.

3. Josephson junction radiometers. - In this section are summarized the results obtained with the three previous versions of the Josephson junction radiometer used at the $2.7 \mathrm{~m}$ McDonald Observatory telescope. In the discussion of the third version we give the values for the radiometer sensitivity $\delta T_{\text {rms }}$ based both on laboratory measurement with hot and cold loads, and based on the signal to noise for an observation of Jupiter.

3.1 Version I : Coudé MOUnTING. - The objective of the first version was to attempt to use a point contact Josephson junction to detect $1 \mathrm{~mm}$ radiation from an astronomical source. A Josephson junction was mounted in a cavity at the bottom of an oversize waveguide leading down the neck of a standard glass laboratory helium dewar. The Coudé focus was chosen because at this focus the large dewar could be fixed in position, and did not have to be moved with the telescope. The waveguide entrance was placed at the telescope focus, and the entering radiation chopped by a reflective metallic chopper that alternately admitted radiation from the telescope or reflected the low temperature thermal radiation from the environment of the junction back to the junction (" total power chopper »). With this radiometer, the $1 \mathrm{~mm}$ thermal radiation from the sun was first-detected on March 31, 1969, and radiation from the moon detected on April 2, 1969 [1]. Thus the first version was useful in determining that it was 
possible to detect $1 \mathrm{~mm}$ radiation from an astronomical object. Some useful astronomy was done in observing enhanced $1 \mathrm{~mm}$ emission from active regions on the sun.

3.2 Version II : Coudé MOUNTING. - The objective for the second version was to improve radiometer sensitivity with a differential chopping system to observe the planets. The detector was still mounted at the Coudé focus because the secondary mirror for the Cassegrain focus of the $2.7 \mathrm{~m}$ telescope had not yet been delivered. The chopper was a partially gold coated mirror that reflected $1 \mathrm{~mm}$ radiation but transmitted $40 \%$ of the visible radiation. The chopper was oscillated about an axis by a loudspeaker drive, thus moving the $1 \mathrm{~mm}$ image of the object on and off the entrance of the waveguide. The chopper transmitted a sufficient fraction of the visible wavelengths that the telescope could be guided visually to track the planet. The voltage output of the preamplifier across the current-biased Josephson junction was converted to a frequency by a voltage-to-frequency converter, and recorded by a multi-channel scaler that was swept through its channels synchronously with the motion of the chopper mirror. With this technique, Venus was detected. However, at the Coudé focus, it would have been difficult to design the required rotating offset guider that would have been necessary for observations of fainter objects, and also it would have been difficult to build a feed horn to match the $\mathrm{f} / 33$ beam from the telescope.

3.3 Version III : CASSEgRain mounting. - The first Cassegrain secondary to be delivered to the 107 inch telescope was the $\mathrm{f} / 18$ secondary. A radiometer was designed and built that incorporated an offset guider and a choice of either a total power chopper, or a differential chopper. With this radiometer, Jupiter was observed. Also there was a possible detection of 3C 273 (3:1 signal to noise ratio), and an upper limit for the galactic center. The results for 3C 273 and the galactic center were reported at the 1971 Liege symposium on infrared astrophysics [2]. The radiometer was also used to measure the limb profile of the sun during the March 1970 partial solar eclipse visible from Texas.

4. Present radiometer (version III) : solid state physics viewpoint. - In the present Josephson junction radiometer for astronomical observations, the wide-band video mode of detection is used. Only this « video " mode of detection is discussed here because it is deemed by the author that only this mode is near to being technically ready for application in astronomy. Other promising modes exist, such as the heterodyne mode. The sensitivity of the present Josephson junction radiometer is limited by questions of technique rather than by the physics of the Josephson junction : the stability of the point contacts is good and is not a present limitation. The electrical noise immunity is poor, and is the present limitation.

4.1 Physics of VIDEo Detection. - «Video » detection is a term borrowed from radar terminology, where it means the detection (rectification) of a microwave signal by a semiconductor diode rectifier. We use the term video detection to mean any microwave detection technique where the output signal is near zero frequency, as opposed to an output signal at an intermediate frequency as in a heterodyne detector. For microwave detection by a semiconductor diode, video detection is simply rectification by the nonlinear current-voltage characteristic of the semiconductor diode. Although the current-voltage characteristic of a Josephson junction is nonlinear, the video detection does not occur simply as a rectification process. Rather, the incoming microwave radiation interacts with the junction via the Josephson equations and modifies the dc current-voltage characteristic. The resulting change in the current-voltage characteristic can be observed, for example, by currentbiasing the junction to a small voltage $(*)$, and by observing the voltage change due to the change in the current-voltage characteristic. In practice, the biasing network for the junction will have some source resistance, $R_{\mathrm{L}}$, and the video detection technique takes the form shown in figure 1.

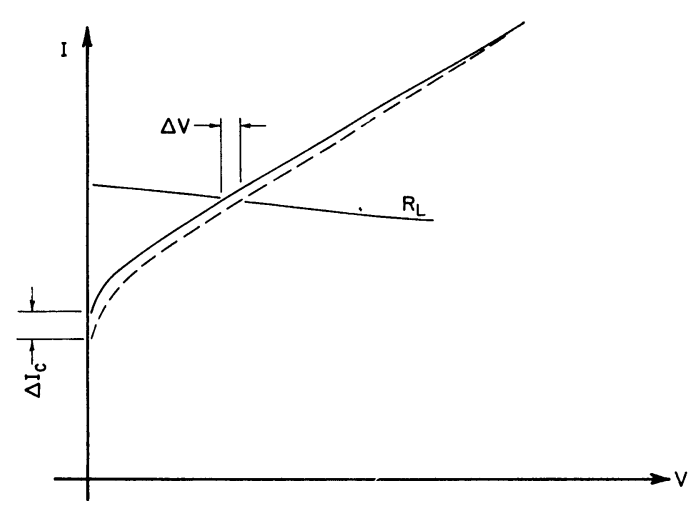

FIG. 1. - Video detection occurs through the change in the $I-V$ characteristic when $1 \mathrm{~mm}$ wavelength is impressed on the junction, rather than through rectification of the signal by the junction. The junction is biased by a load resistance $R_{\mathrm{L}}$, and the change in bias voltage $\Delta V$ due to the changed $I-V$ characteristic is amplified as the output signal. For nearly zero voltage bias, $\Delta V$ is proportional to the change in junction critical current $\Delta I_{\mathrm{c}}$.

Thus this wideband video detection technique presently used is the same as that first used by Grimes, Richards and Shapiro [7]. The voltage change due to a change in the zero voltage current of a junction dc biased by a source resistance $R_{\mathrm{L}}$ is measured with a lock-in amplifier at the chopping frequency. It can

(*) A small voltage is one that is small compared to the bias voltage that would give an ac Josephson current at the microwave signal frequency. Thus, we must have $V_{\text {Bias }} \ll V_{\mathrm{J}}=\hbar \omega / 2$ e. 
be shown from the voltage-biased model of the Josephson junction that voltage change across the junction for an incident power $P$ at the frequency $\omega$ is approximately

$$
V=\frac{I_{0} Z R_{\mathrm{D}} R_{\mathrm{L}}}{2\left(R_{\mathrm{D}}+R_{\mathrm{L}}\right)}\left(\frac{2 \mathrm{e}}{h \omega}\right)^{2} P
$$

where $R_{\mathrm{D}}$ is the dynamic dc resistance of the junction current-voltage curve, and $Z$ is an effective input impedance for the junction. An improved model for the current biased junction was developed by Kanter and Vernon [8] and the predicted spectral response verified by Blaney [9]. The result of Kanter and Vernon applies especially to the case where a junction is current biased to a voltage corresponding to the frequency under observation, and the voltage change due to the incident radiation is detected. When the change of the zero voltage current is detected, the intrinsic spectral response of the junction depends on frequency as $1 / \omega^{2}$. As observed by Grimes, Richards and Shapiro [7], the actual response is determined primarily by the frequency dependent coupling of the metallic structure surrounding the junction.

4.2 OTHER MODES OF DETECTION. - As noted above, a promising mode of detection for the future is heterodyne detection with either internal (ac Josephson oscillation) or external local oscillator. However, only the wide band video mode of detection appears ready for immediate development for use in an astronomical instrument. The video mode offers high sensitivity for continuum observations and should be developed even if a successful heterodyne detector is developed.

4.3 TeChNiques OF VIDEO DETECTION. - 4.3.1 Stability of the point contact. - Point contact Josephson junctions are used in the present radiometer because they couple successfully to the $1 \mathrm{~mm}$ radiation and because they have high sensitivity as described in the astronomical techniques section of this paper. The stable point contacts that have been developed withstand cycling between helium and liquid nitrogen temperatures for several days. Results for such a test are shown in figure 2, which illustrates the junction current-voltage curves. The change in critical current over four days was $10 \%$. The sensitivity decreased over the same period by a factor of two, but this change may have been due to ice formation on the inside of the light pipe leading to the junction. The standard technique to test for point contact stability is to rap on the side of the dewar with the knuckles. Not all point contacts are stable, but it is nearly always possible to obtain a point contact that is sufficiently stable. Less than $20 \%$ of the observing time has been lost due to mechanical instability of the point contacts. The most important technique which has been used to ensure that the contacts are stable is to make the differential screw junction mount rigid, and to isolate it from external vibrations. In general, the mechanical stability of the point contacts is not a problem.

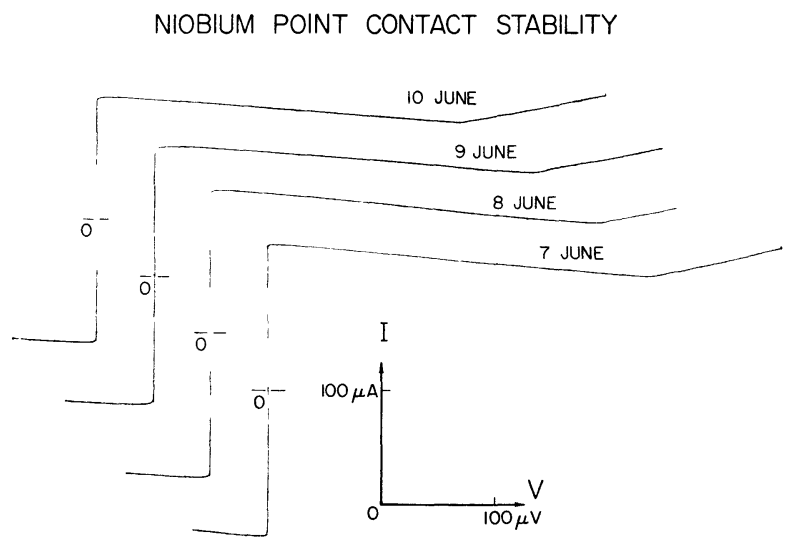

Fig. 2. - Current-voltage curves on successive days were taken to study the stability of a junction formed by a niobium point on tin. The point contact was not adjusted between measurements. After each day the junction warmed up to liquid nitrogen temperature $(77 \mathrm{~K})$, and liquid helium was retransferred into the dewar. The stable negative resistance region is due to parametric oscillations.

4.3.2 Electrical noise immunity. - The greatest present technical problem is in making the junction immune to ambient electrical noise. On occasion, military radar near the observatory and Radio Netherlands have been picked up, as well as observatory generated noise. The junction is a wideband detector from dc to at least $3 \times 10^{11} \mathrm{~Hz}$. This result follows directly from the expressions giving the junction sensitivity. The present radiometer system uses a glass dewar that is not immune to noise. An all-metal dewar is finished. It holds 21 of helium $27 \mathrm{~h}$ without a nitrogen jacket. A Josephson junction mount and the adaptation to the differential chopping system remain to be built for it.

5. Present radiometer (version III) : astronomical viewpoint. - 5.1 SENSITIVITY MEASURED IN THE LABORATORY. - The sensitivity has been measured in two ways : with hot and cold loads, and by observing Jupiter.

An optical diagram of the present (version III) radiometer is shown in figure 3 . The focus of the telescope is near the plane of the offset guider. The focal point is re-imaged by an $\mathrm{f} / 0.3$ off-axis paraboloid onto the entrance of the waveguide after reflection from the differential chopper mirror. The waveguide is on the axis of the paraboloid. The purpose of the paraboloid is to convert the $\mathrm{f} / 18$ beam from the telescope secondary to the $f / 2$ beam accepted by the oversize waveguide. Just above the waveguide entrance is the total power reflective chopper which alternately opens and closes the waveguide at $300 \mathrm{~Hz}$.

The differential chopper is a plane mirror that can be oscillated at $37 \mathrm{~Hz}$ about the axis of the paraboloid 


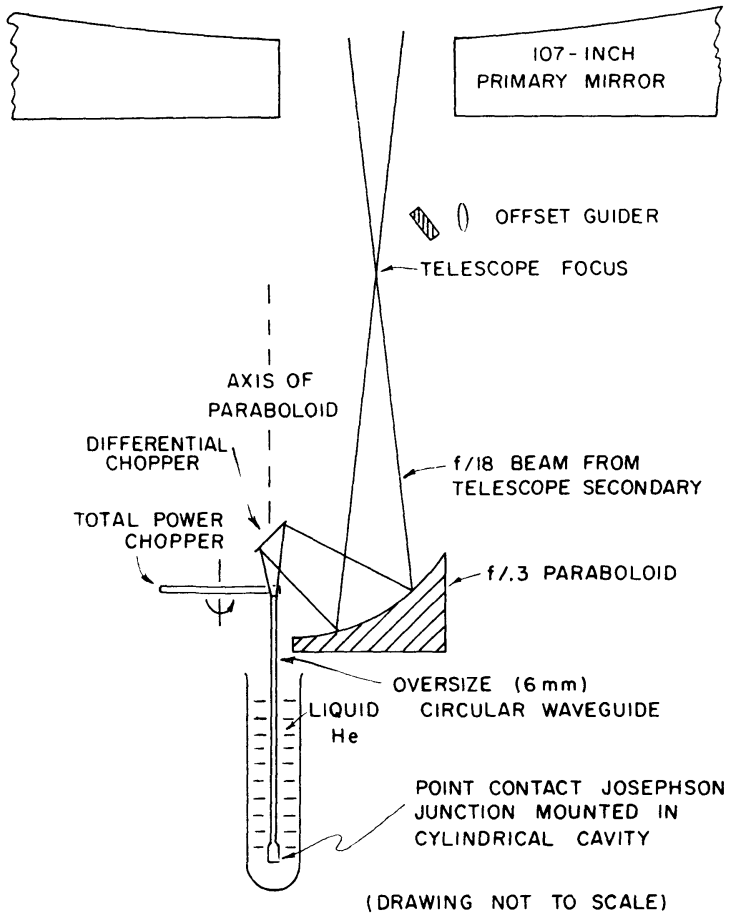

FIG. 3. - Optical diagram of the version III Josephson junction radiometer mounted at the $\mathrm{f} / 18$ Cassegrain focus of the $2.7 \mathrm{~m}$ (107 inch) optical telescope.

to move the image of the object on and off the waveguide entrance. The total power chopper can be swung out of the way when the differential chopper is being used, and the differential chopper can be turned off when the total power chopper is being used.

To measure the sensitivity $\left(\delta T_{\mathrm{rms}}\right)$ of the radiometer, the total power chopper was used, and a hot or cold load was placed at the image of the waveguide entrance at the focal plane of the telescope. The hot load was foam microwave absorber (Eccosorb) at ambient temperature. The cold load was the same foam immersed in a polystyrene coffee cup filled with liquid nitrogen. The output of the lock-in amplifier that demodulated the chopped signal was converted to a frequency, and counted for $1 \mathrm{~s}$ intervals in a multichannel scaler, as shown in figure 4 .

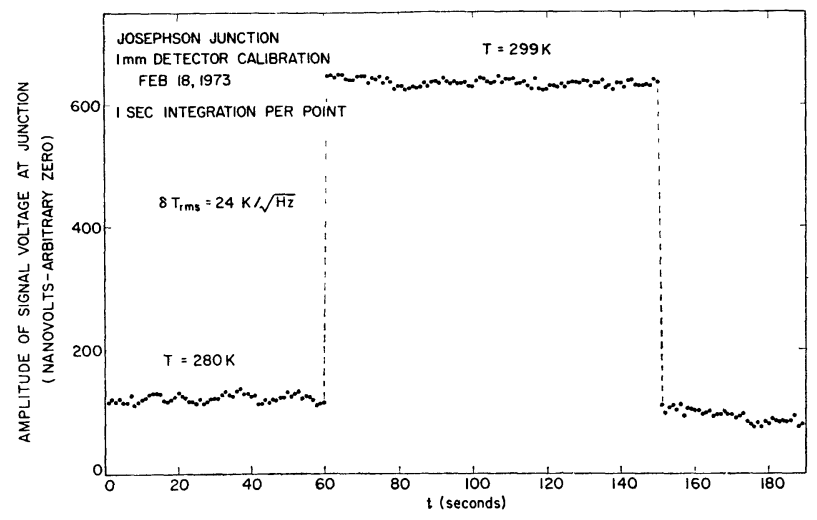

FIG. 4. - Calibration of the Josephson junction $1 \mathrm{~mm}$ wavelength radiometer, using hot and cold loads, placed at the focus at the entrance to the radiometer.
From these data the sensitivity

$$
\delta T_{\mathrm{rms}}=0.25 \mathrm{~K}
$$

for a 1 s observation.

5.2 ObSERVATIONS OF JUPITER. - The best test of he sensitivity of the present system is to determine ne signal to noise during an observation of an astronomical object. For this purpose, Jupiter was observed. The semidiameter of Jupiter was 19.5". The differential chopper scanned the image of Jupiter 37 times a second on and off the entrance to the waveguide leading to the detector. The $37 \mathrm{~Hz}$ signal was detected by a digital lock-in technique as follows. As before, the output of the voltage preamplifier connected to the current biased junction was connected to a voltageto-frequency converter. The output pulses were counted in a multichannel scaler (Technical Measurement Corporation) in which the channel was advanced every millisecond. The multichannel scaler was cycled through its memory once each cycle of the differential chopper. Thus the scaler built up more counts when the Jupiter image was on the waveguide entrance. The shape of the pattern obtained by scanning Jupiter on and off the detector was determined by the diffraction spot of the beam leaving the telescope. Actually, in addition to scanning Jupiter on and off the detector, the differential chopper also scanned a background signal from the telescope on and off the detector. This background signal was due to asymmetric sidelobes of the oversize waveguide which saw parts of the telescope that did not appear to be symmetric about the axis of the waveguide. Thus, a large asymmetry signal was built up.

This background asymmetry signal was cancelled in the following way : the differential chopper scanned the telescope in a north-south direction. Data was first collected with Jupiter in the north beam, and counts were added in the multi-channel scaler. Then the telescope was moved to place Jupiter in the south beam and counts were subtracted in the multichannel scaler. This move did not affect the background asymmetry signal. The synchronization between the chopper and the memory advance of the scaler was kept constant. In this way, the telescope asymmetry signal was first added and then subtracted for equal lengths of time. However, the differential signal from Jupiter was first added, and then when Jupiter was moved to the south beam, the sign of the signal changed but was subtracted in the scaler. The net result was that the signal added both times, but the background asymmetry was cancelled out. The result of this procedure is shown in figure 5. The upper part of the figure illustrates the signal from Jupiter ; the lower part shows the degree to which the telescope asymmetry signal can be cancelled for a "blank » region of sky several beam widths away from Jupiter. The signal amplitude for Jupiter was determined by a maximum-likelihood fit of a sine function to the 
observed signal versus differential chopper position. The signal to noise for Jupiter is $80: 1$ for an $8.8 \mathrm{~min}$. integration $(4.4 \mathrm{~min}$. in the north beam and $4.4 \mathrm{~min}$. in the south beam).

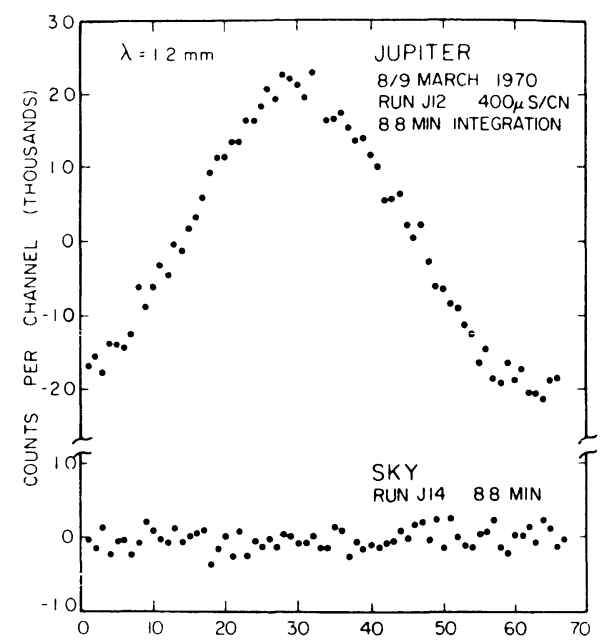

FIG. 5. - Observation of Jupiter with the Josephson junction $1 \mathrm{~mm}$ radiometer, using the $2.7 \mathrm{~m} \mathrm{McDonald}$ Observatory telescope. The degree to which the sky and telescope background signal can be cancelled is shown by the lower portion of the figure.

In principle, the signal to noise for the Jupiter observation can be used to determine a $\delta T_{\text {rms }}$ for the detector. For such a determination, it is necessary to know the brightness temperature of Jupiter at $1 \mathrm{~mm}$, the shape and width of the antenna pattern of the 107 inch telescope at $1 \mathrm{~mm}$, and the aperture efficiency of the telescope. The first two parameters can be estimated or measured. The $1 \mathrm{~mm}$ brightness temperature of Jupiter can be extrapolated from measurements at longer wavelengths, and is found

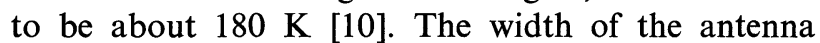
pattern is discussed in the next paragraph. The third parameter, the 107 inch telescope aperture efficiency, is not known at all. Consequently, the observations of Jupiter together with the hot and cold load measurements of the $\delta T_{\text {rms }}$ will be used to determine the aperture efficiency of the $2.7 \mathrm{~m}$ telescope, as it is coupled to the Josephson junction radiometer.

To determine the width of the antenna pattern, which is needed to calculate the antenna temperature of Jupiter, the telescope was scanned across the limb of the sun $15 \mathrm{~h}$ before the observation of Jupiter, as shown in figure 6 . The total power mode of chopping was used. It was assumed that the limb of the sun is a step function at one millimeter compared to the telescope beam width. This assumption can be justified from observations with the Josephson junction radiometer during the March 1970 partial solar eclipse visible from Texas: Within $20 \%$ accuracy there is neither limb brightening nor limb darkening at $1 \mathrm{~mm}$ [11]. The scan across the east-west diameter of the sun is shown in figure 6. From the slope

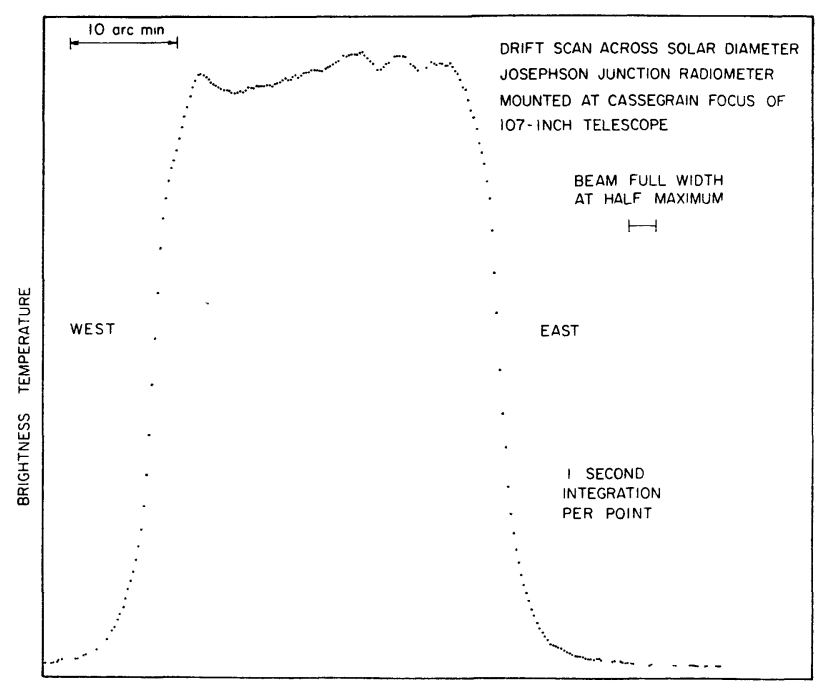

FIG. 6. - Drift scan across East-West diameter of the sun with the Josephson junction $1 \mathrm{~mm}$ radiometer in the total power mode of chopping, showing structure due to solar active regions. The noise level is indicated in the data to the East of the sun.

at half maximum, the data at the limb of the sun were fit to a gaussian shaped antenna pattern, $I=I_{0} \exp \left(-\theta^{2} / \theta_{0}^{2}\right)$, with $\theta_{0}=1.6^{\prime}$. The Full Width Half Maximum (FWHM) beamwidth is $2.6^{\prime}$. A uniformly illuminated annulus of 107 inch outside diameter with a 50 inch diameter central obstruction has a FWHM beam of $1.4^{\prime}$, at $1.2 \mathrm{~mm}$ wavelength. At the present time the excess antenna beamwidth is attributed to the way in which the antenna is illuminated by the oversize waveguide and the f-ratio transforming paraboloid. It is not known what modes of the oversize waveguide couple to the junction and thus illuminate the primary mirror. There is, however, no correction for errors in the figure of the mirror at one millimeter, because the primary and secondary mirror are of visual wavelength quality.

For a point source, the telescope aperture efficiency $\eta$ is defined by the relation

$$
T_{\mathrm{A}}=\eta A_{\mathrm{p}} \frac{S}{2 k}
$$

Where $T_{\mathrm{A}}$ is the antenna temperature, $A_{\mathrm{p}}$ is the physical area of the telescope based on the full diameter of the telescope, and neglecting the obstruction by the secondary, $S$ is the flux from the radio source in $\mathrm{W} \cdot \mathrm{m}^{-2} \cdot \mathrm{Hz}^{-1}$ and $k$ is Boltzmann's constant. When the source is extended as is Jupiter, it is necessary to integrate over the solid angle subtended. Assuming the gaussian beam shape as before, and a uniform temperature $T_{\mathrm{J}}$ over the disk of Jupiter, we find

$$
T_{\mathrm{A}}=\eta A_{\mathrm{p}} \frac{\pi \theta_{0}^{2}}{\hat{\lambda}^{2}}\left[1-\exp \left(\frac{-\theta_{\mathrm{J}}^{2}}{\theta_{0}^{2}}\right)\right] T_{\mathrm{J}} .
$$


When the source is observed for a time $t_{\text {obs }}$, the signal to noise ratio $Y$ will be

$$
Y=\frac{T_{\mathrm{A}}}{\delta T_{\mathrm{rms}}}\left(\frac{t_{\mathrm{obs}}}{1 \mathrm{~s}}\right)^{1 / 2} .
$$

The last two expressions may be combined to yield the aperture efficiency

$$
\eta=\frac{Y \delta T_{\mathrm{rms}}}{A_{\mathrm{p}} T_{\mathrm{J}}} \frac{\lambda^{2}}{\pi \theta_{0}^{2}} \frac{1}{1-\exp \left(-\theta_{\mathrm{J}}^{2} / \theta_{0}^{2}\right)}\left(\frac{1 \mathrm{~s}}{t_{\mathrm{obs}}}\right)^{1 / 2}
$$

which upon substitution of the values for the observation of Jupiter at the 107 inch telescope gives an $\eta$ of 0.09 for the aperture efficiency at $1.2 \mathrm{~mm}$ wavelength. The $\delta T_{\text {rms }}$ used in the expression was obtained with hot and cold loads while the detector was mounted on the 107 inch telescope as shown in figure 3. Such a low aperture efficiency is attributed to difficulties in illuminating the primary mirror.

5.3 SENSITIVITY IN FLUX UNITS AT THE 107 INCH AND 36 FOOT TELESCOPES. - From the observations of Jupiter at the 107 inch telescope, and the aperture efficiency derived from these observations, we can derive the sensitivity in flux units of the present radio- meter (version III) at the 107 inch telescope from the relation

$$
\delta S_{\mathrm{rms}}=\frac{2 k \delta T_{\mathrm{rms}}}{\eta A_{\mathrm{p}}}
$$

which gives

$$
\delta S_{\mathrm{rms}}=\frac{2 \times 1.38 \times 10^{-23}}{0.09 \times 5.8} \times \underline{0.25}=1300 \mathrm{fu}
$$

for a $1 \mathrm{~s}$ observation. If the present observing system were taken and mounted at the Cassegrain focus of the 36 foot telescope, then the sensitivity would be

$$
\delta S_{\mathrm{rms}}=\frac{2 \times 1.38 \times 10^{-23} \times 0.25}{0.1 \times 94.6}=73 \mathrm{fu}
$$

for $1 \mathrm{~s}$ observation where we have used an $\eta$ of 0.1 and an $A_{\mathrm{p}}$ of $94.6 \mathrm{~m}^{2}$. For a $3 \mathrm{~h}$ observation, the noise would be $0.7 \mathrm{fu}$. The above estimates assume a $50 \%$ chopping duty factor, which was the case during the measurement of the $\delta T_{\mathrm{rms}}=0.25 \mathrm{~K} / \sqrt{\mathrm{Hz}}$.

The noise equivalent power (NEP) is not directly relevant to observing time estimates for continuum sources. It is difficult to estimate the NEP because information on the bandwidth of the detector is

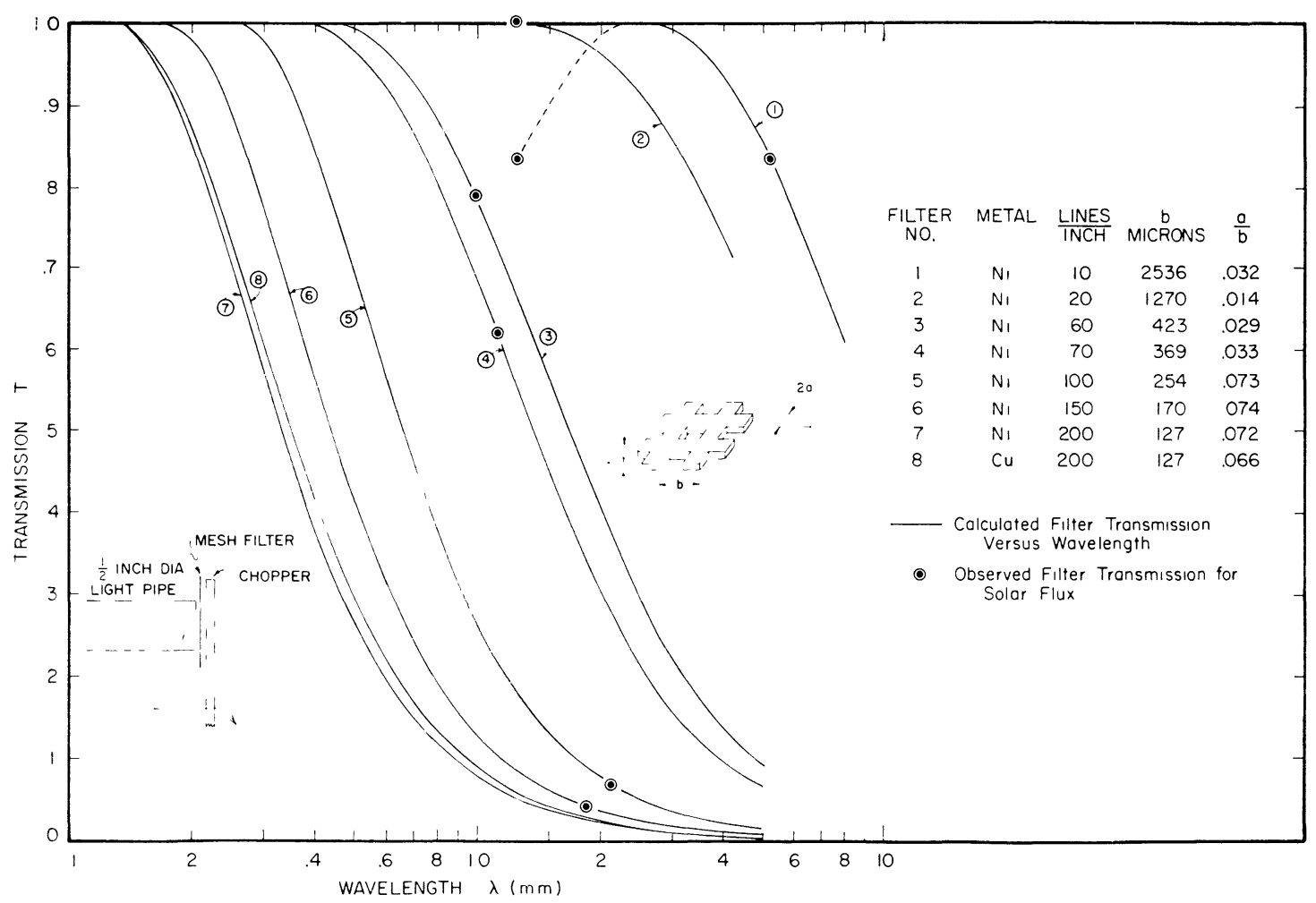

FIG. 7. - Calibration of the effective wavelength of the Josephson Junction $1 \mathrm{~mm}$ wavelength radiometer. Metal mesh filters were used as short wavelength pass filters. The solid lines show the monochromatic filter transmission versus wavelength. The filters were interposed between the total power reflective chopper and the entrance to the Josephson junction radiometer while the telescope was pointed at the sun, and the apparent transmission of each filter was measured. The points indicate at what wavelength a monochromatic source would give the observed filter transmission. The actual detector response is given by an integral over the detector response versus wavelength, the atmospheric transmission, and the energy distribution of the sun versus wavelength. The measurements indicate a radiometer effective wavelength between 1 and $2 \mathrm{~mm}$ for the solar spectral energy distribution. The point for curve 1 falls on the short wavelength side of the peak filter transmission, where diffraction reduces the transmission. The diffraction region of the filter transmission has not been calculated, but has been sketched in by the dotted line. 
lacking. One of the objectives of present plans is to provide the spectral sensitivity information needed to estimate the NEP.

5.4 EfFective WAVELENGTH. - The effective wavelength of the junction used in this broadband mode of detection was measured with a set of metal mesh filters which were interposed between the total power chopper and the waveguide entrance. Each filter transmits wavelengths shorter than about the grid spacing, and reflects longer wavelengths. The theoretical transmission versus wavelength is shown by the solid lines in figure 7 . If the junction response were nearly monochromatic, then the transmission of each filter would give the wavelength at which the Josephson junction is sensitive. The actual transmission is the convolution of the filter transmission with the junction sensitivity. The measured transmissions for the set of filters give " monochromatic wavelengths" that cluster between 1 and $2 \mathrm{~mm}$, but do not determine an accurate value of the effective wavelength. Also, the measurement is not precise enough to determine the detector bandwidth which would be necessary to convert the $\delta T_{\text {rms }}$ sensitivity into a noise equivalent power. However, the $\delta T_{\text {rms }}$ is the directly useful quantity for determining what continuum radio sources can be observed with the detector.
5.5 Limitations IN THE PRESENT SENSITIVITY. These are as follows :

1) External electrical noise. As discussed above, this noise in the past has often limited the radiometer signal to noise ratio, but will be much less serious with the metal dewar.

2) Amplifier noise. The present preamplifier uses a room temperature transformer to match impedances and a room temperature amplifier. Amplifier noise dominates on the occasions when the junction responsivity is low.

3) Junction noise. On some occasions when the junction responsivity is high, noise is seen that is higher than amplifier noise. No study has been made of this noise, and it is not known whether it is the intrinsic noise of the junction [12] or whether it is noise coming from the amplifier input or coming from some other external source that is then rectified by the junction.

Acknowledgments. - We thank the staff of McDonald Observatory and especially Laughlin, C., Dittmar, D., Harvey, F., Grobar, J., Cobb C. and Liddell, J. for their assistance in marking this work possible. We thank the National Science Foundation for support through NSF-USDP GU-1598.

\section{References}

[1] Ulrich, B. T., "Superconducting Link Electromagnetic Detectors : An application of the Josephson Effect », in Cryogenics and Infrared Detection Systems ((W. H. Hogan and T. S. Moss, editors, Cambridge : (Boston Technical Publishers)), 1970, p. 120-137.

[2] Ulrich, B. T., « $1 \mathrm{~mm}$ Observations of 3C 273 and the Galactic Center with a Josephson Effect Receiver », in Les Spectres des Astres dans L'Infrarouge et les Microondes, Mémoires de la Société Royale des Sciences de Liège, Sixième Série, Tome III, 1972, p. 337.

[3] Ulrich, B. T., "Josephson Junctions in Astronomy », in Proceedings of the Twelfth International Conference on Low Temperature Physics (E. Kanda, editor, Kyoto: Keigaku Publishing Co.), 1971, p. 867.
[4] Kellermann, K. I., Pauling-Toth, I. I. K., Astrophys. Lett. 8 (1971) 153.

[5] Kellermann, K. I., Private communication.

[6] Beckman, J. E. and Clark, C. D., Solar Physics 16 (1971) 87.

[7] Grimes, C. C., Richards, P. L. and Shapiro, S., Phys. Rev. Lett. 17 (1966) 431.

Grimes, C. C., Richards, P. L. and Shapiro, S., J. Appl. Phys. 39 (1968) 3905.

[8] Kanter, H., Vernon, F. L., J. Appl. Phys. 43 (1972) 3174.

[9] Blaney, T. G., Phys. Lett. 37A (1971) 19 ;

BlaNEY, T. G., Radio and Electronic Engineer 42 (1972) 303.

[10] Cogdell, J., Private communication.

[11] Ulrich, B. T., Unpublished.

[12] Lee, Patrick, A., Ph. D. Thesis, Mass. Inst. of Technology. 